Comparative Philosophy Volume 6, No. 2 (2015): 58-66

Open Access / ISSN 2151-6014

www.comparativephilosophy.org

\title{
EDITOR'S POSTSCRIPT: FROM THE VANTAGE POINT OF CONSTRUCTIVE-ENGAGEMENT STRATEGY OF COMPARATIVE PHILOSOPHY
}

The current issue (volume 6, no. 2, July 2015) is a special-topic issue on "Crosstradition Engagement in Philosophy: Meta-Philosophical and Meta-Methodological Reflections". The topic as a whole highlights one central concern of comparative philosophy and thus one of the major concerns of this journal, on meta-philosophical and meta-methodological exploration of how cross-tradition engagement in philosophy is possible, which addresses general theory and methodology of comparative philosophy (or doing philosophy "comparatively"). The discussion as presented in the current special-topic issue is especially relevant and engaging because of this: to have this journal with due focuses and emphases in the current prolific philosophy journal market and in view of the reflective need in the area, this journal is guided (in such aspects as submission topic and emphases, peer-review vantage point, etc.) by a general "constructive-engagement" strategy of comparative philosophy, as elaborated in the journal-theme-introduction essay. ${ }^{1}$ This general approach is an "open" strategy in two connections as suggested by its name. First, on the one hand, it is "constructively" open: it looks at distinct views on the issue of how cross-tradition engagement in philosophy is possible for a "constructive" purpose and in a "constructive" way (how we can learn from each other and jointly contribute to the common goal of contemporary development of philosophy and the development of contemporary society). Second, on the other hand, it is "open" in an engaging way: through criticism and self-criticism and philosophical argumentation to implement the foregoing "constructive" goal and way, instead of taking a passive and lessphilosophically-interesting "mere-tolerance" attitude. This "open" character brings about some organizational strategies of the journal content: besides those that appear in the regular "Article" parts, special sessions that explicitly generate such engaging discussions are also given in such forms as "constructive-engagement dialogue", "the special-topic issue", etc. This "Editor's postscript" is intended to have the foregoing "open" character and provide engaging commentary on some of interesting points made in the contributing articles from the vantage point of the constructiveengagement strategy of comparative philosophy, though this writing as an engaging

\footnotetext{
${ }^{1}$ Mou, Bo (2010), "On Constructive-Engagement Strategy of Comparative Philosophy: A Journal Theme Introduction", Comparative Philosophy 1.1: 1-32<http://www.comparative philosophy.org>.
} 
commentary is not intended to be comprehensive and formal but selective in an "informal" style: I will not repeat all those important points that have been already well presented in the authors' articles; rather I will focus only on some of them on which I intend to have a number of substantial "critical" comments, more or less, instead of "celebrating" ones that have been already suggested as they were selected to appear in this journal through various channels of peer-review process.

One more note on the subtitle of this writing 'From the vantage point of constructive-engagement strategy of comparative philosophy'. Though the above briefly explains how this strategy as a meta-meta-philosophy/methodology attitude is relevant and engaging here, there is another interesting aspect in which the "constructive-engagement strategy" is addressed in this postscript writing. It is not just a strategic attitude but also gives a working meta-methodological framework for evaluation and constructive engagement: the relevant resources can be used for the sake of effective evaluation and thus can be tested for their explanatory force. In my subsequent engaging comments, there is the need to reiterate certain relevant conceptual resources introduced earlier (in the journal-theme-introduction article) in order to refine understanding and have effective evaluation: among others, the distinction between methodological perspectives, methodological instruments, and methodological guiding principles, together with some implied methodological morals. It is known that the term 'method' or 'methodological approach' in philosophical inquiries can mean a number of things. Given that the term 'methodological approach' means a way responding to how to approach an object of study, without due clarification and differentiation, the term might be used to mean one of the following three methodological ways or some of them simultaneously: a methodological perspective (or a perspective method), a methodological instrument (or an instrumental method), and/or a methodological guiding principle (or a guidingprinciple method). (1) Roughly speaking, a methodological perspective is a way of approaching an object of study and is intended to point to or focus on a certain aspect of the object and capture or explain that aspect in terms of the characteristics of that aspect, together with the minimal metaphysical commitment that there $i s$ that aspect of the object or that the aspect is genuinely (instead of being merely supposed to be) possessed by the object. There is a distinction between eligible and ineligible methodological perspectives concerning an object of study. If the aspect to which a methodological perspective is intended to point is genuinely possessed by the object of study, it is considered eligible in regard to that object. Otherwise, the methodological perspective is considered ineligible in regard to that object. $^{2}$ (2) A methodological instrument is a way in which to implement, or give tools to realize, a certain methodological perspective. (3) A methodological guiding principle regulates

${ }^{2}$ It is noted there is the distinction between a methodological-perspective simplex and a methodological-perspective complex. A simplex is a single discernible methodological perspective, and a complex is either a combination of simplexes ("multiple perspective complex") or an association of one perspective (simplex) with a certain methodological guiding principle ("guiding-principleassociated perspective complex"). By "perspective" below I mean a methodological perspective simplex unless otherwise indicated. 
and guides a certain methodological perspective (or perspectives) in regard to an object of study; presupposed by the agent, it implicitly guides and regulates how the perspective should be evaluated and used and contributes to the establishment of its desiderata (especially, the purpose and focus that it is to serve). There is the distinction between adequate and inadequate methodological guiding principles concerning methodological perspective(s) in regard to an object of study. Furthermore, I would like to call the readers' attention to the adequacy conditions as given in the theme-introduction article, which together with the related resources suggests a meta-methodological framework by which to carry out the constructive engagement and evaluate its result. Given the above specifications, there are two preliminary points concerning the relation between a methodological perspective and a methodological guiding principle, which are especially relevant to the subsequent discussion. First, generally speaking, the merit, status, and function of a methodological perspective per se can be evaluated independently of a certain methodological guiding principle that the agent might presuppose in her actual application of the perspective. One's reflective practice per se of taking a certain methodological perspective as one's working perspective amounts to neither one's losing sight of other genuine aspects of the object nor one's rejecting other eligible perspectives in one's background thinking nor presupposing an inadequate methodological guiding principle which would render ineligible other eligible methodological perspectives (if any). Second, however, it does matter whether one's taking a certain methodological perspective is regulated by an adequate or inadequate guiding principle, especially for the sake of constructive engagement of seemingly competing approaches. When one's application of an eligible methodological perspective as part of one's reflective practice is guided by an adequate guiding principle and contributes to adequate understanding of the object of study, one's application of that perspective would be philosophically constructive.

1. I have two major comments on Justin Smith's article "Tradition, Culture, and the Problem of Inclusion in Philosophy". Smith correctly points out Hegel's evaluative attitude toward the identity of philosophy: it seems to Hegel, "[p]hilosophy comes into being in earnest when it becomes autonomous from the cultural life of its practitioners, and this in turn is possible only under historical conditions, such as those that first obtained in classical Greece, in which men experience themselves as free individuals." [5]. ${ }^{3}$ It is important to note that Hegel's foregoing evaluative attitude is concerned with how to evaluate the relation between the autonomous approach in philosophical studies and the identity of philosophy, and this view is part of the "methodological guiding principle" dimension of his approach as a whole, which is distinct from the "perspective" dimension of his approach in which an "autonomous" perspective per se is taken. Smith also correctly identifies what is shared by Hegel, Gilbert Harman, and Margaret Wilson under his examination: "Harman and Hegel are in fact not so far apart: both take philosophy to be an

\footnotetext{
${ }^{3}$ The page numbers of the three authors' citation references in the current issue are given in bracket parentheses.
} 
autonomous discipline, unconnected to the study of culture. Wilson is not so far from them either: she recognizes that one has to engage with the efflorescences of culture not just "foreign" languages, but also, when necessary, literary forms such as the philosophical novel or poem-but only as a means to the end that she shares with Harman and Hegel alike: autonomous engagement with philosophical ideas.'[6] That is, what they share is the "autonomous" perspective in philosophical exploration, although they have their distinct "guiding principles" on how to look at the status and nature of this working perspective in view of other eligible perspectives. Now what is at issue is how to look at and evaluate the status of the autonomous approach per se as one perspective that is taken by many to be their working perspective in philosophical studies. My view is this. The merit, status, and function of the "autonomous" perspective per se can be evaluated independently of a certain methodological guiding principle that an agent (say, Hegel, Harman, ${ }^{4}$ or Wilson) might presuppose in her actual applications of the perspective; one's reflective practice per se of taking the "autonomous" methodological perspective to look at the "autonomous" aspect (if any) of an object of study (as one's working perspective) amounts to neither one's losing sight of other genuine aspects of the object nor one's rejecting other eligible perspectives in one's background thinking nor presupposing an inadequate methodological guiding principle which would render ineligible other eligible methodological perspectives (if any). In this way, I think there is the need to be careful about exactly which part of Hegel's approach under examination goes wrong: generally speaking, there is nothing wrong with taking the "autonomous" perspective per se when this perspective in looking at a certain object of study is eligible, even it can be expected and in need when one's purpose and focus is on the "autonomous" aspect of the object; specifically speaking, there is nothing wrong with the "autonomous" perspective per se when it is used to point to the "autonomous" aspect that would be genuinely possessed by philosophical exploration and thus with the "autonomous" perspective dimension of Hegel's approach as a whole; what is problematic lies in its treating the autonomous expectation as the exclusive or absolutely superior one. In other words, whether or not its "methodological guiding principle" dimension is adequate (arguably inadequate), its "perspective" dimension can be still eligible; whether or not a certain perspective can be taken to be one (eligible) working perspective in treating an object of study would be sensitive to

\footnotetext{
${ }^{4}$ Based on my own first-hand experience, I would like to say that, though persistently maintaining the "autonomous" perspective as his working perspective in his own philosophical exploration, Harman's methodological guiding principle concerning how to look at the status of some other "perspective" approaches from other philosophical traditions in view of its relation to his own "autonomous" perspective seems to change in recent years. In the 2011 summer, I participated in Harman's and Ernie Lepore's jointly directed NEH Summer Seminar on Quine and Davidson at Princeton University. In a post-session workshop I presented a paper draft on how the constructive engagement between Quine's naturalized epistemology and Zhuang Zi's naturalism is possible; Harman not only attended this postsession workshop, whose participation was not requited, but was also interested in the content of the paper and gave comments on scene. [The revised version of this paper was published in The Philosophical Challenge from China, edited by Brian Bruya (MIT Press; 2015), 303-337.]
} 
one's purpose and focus, to the context in which one's reflective interest counts and to whether the object of study really possesses the aspect to which the perspective points and captures.

My second major comment on Smith's article is concerned with the author's ending point calling people to recognize that "the study of philosophy is, among other things, the study of culture" [7]. This dictum, on the one hand, hits the author's point home to some extent, and on the other hand its expression as given above can be misleading. To be fair to the author, though using the period to end that dictum, Smith immediately has one substantial modification of the foregoing dictum: "Such a transformation, I maintain, is a necessary precondition of any future form of academic philosophy that will satisfy the current desideratum of greater inclusiveness." (ibid., my emphasis in italics). The dictum reads more reasonable under this modification: that is, it is so when one takes a certain perspective, —call it a "culture" perspective concerning greater inclusiveness; my question here is then this: to have this claim nontrivial, one needs to first specify what is meant by 'culture' for the sake of greater inclusiveness: if it just means, say, a certain culture-bound natural language by which to linguistically express one's philosophical thought, the statement is certainly trivially right; however, I believe the author means something more than mere culture-bound language but something non-linguistically cultural; then one might ask: when one's focus is on some "non-cultural" aspect of the object of study that is in nature irrelevant to various specific cultural elements and thus takes an "autonomous" perspective, why does one then need to be indiscriminately expected to treat the mentioned "study of culture" as "a necessary precondition" for one's reflection on that aspect even if one intends to include the relevant contributions from other philosophical traditions? I think that this would be one connection in which the author might consider further substantially refining his point.

2. In her article "It's not Them, it's You: A Case Study Concerning the Exclusion of Non-Western Philosophy?", Amy Olberding makes her interesting and engaging challenge which begins with the following thoughtful observation and evaluative remarks [14-15]:

There has of late been increasing attention in professional philosophy to the need to better include non-western philosophies in our discourse....At least some of the difficulty in incorporating non-western philosophy into western-dominated discourses results from a kind of double bind. To gain inclusion, non-western philosophies need effectively to hook in to existing issues, interests, or paradigms in the dominant discourse; non-western philosophies need to offer something that addresses the concerns and preoccupations western-trained philosophers find compelling and significant. However, where these philosophies do successfully hook in, this alone is rarely sufficient. Those scholars, for example, seeking to draw Buddhist no-self doctrines into contemporary discourses may be enjoined to explain just what Buddhist sources offer that Hume or Parfit do not already afford. Scholars working on virtue ethical interpretations of Confucian sources will need to demonstrate what the Confucians can philosophically accomplish that Aristotle cannot. Such is to say that even where non-western philosophical sources share common ground with western philosophies, that ground may yet be treated as fully occupied without them. 
They will simply seem surplus to requirements....Failure to hook in to existing domains of inquiry or doing so in an unfamiliar style may awaken suspicion that here is something other than philosophy proper. The double bind for scholars who would promote interest in non-western traditions, then, can register as an importunate, impossible demand: Show us something we have not seen before, but be sure it looks well and truly familiar to us too. [My emphases in italics.]

It is known that the constructive engagement strategy emphasizes the need to constructively engage on jointly concerned issues in philosophy; the foregoing cited remarks appear to also challenge this strategy in comparative philosophy in this connection to some extent: isn't it that the constructive engagement strategy presupposes the "existing" identities of the issues? The constructive-engagement strategy has already had an explicit answer to such a question, as indicated in one of the suggested adequacy condition for methodological guiding principles (i.e., the new-eligible-perspective-possibility-recognizing condition) $)^{5}$ :

The new-eligible-perspective-possibility-recognizing condition: A methodological guiding principle is considered adequate (in this connection) if it guides the agent to have an openminded attitude toward the possibility of a new eligible perspective that is to point to some genuine aspect of the object of study but has yet to be realized by the agent because of the 'unknown-identity' status of that aspect. A methodological guiding principle is considered inadequate (in this connection) if otherwise.

Clearly, from the vantage point of the constructive-engagement strategy, the identity of a joint-concerned issue in philosophy is not determined by their "existing domain of inquiry" in philosophical studies: the constructive engagement strategy does not take for granted the "existing" identity of what count as the jointly-concerned issues in philosophy: it resorts to neither the current agents' subjective preferences nor the identity of "existing domain of inquiry"; rather, as highlighted in the foregoing "neweligible-perspective-possibility-recognizing" condition for the adequacy of methodological guiding principles in this connection, it has positively suggested some "objective" criterion for the identities of such issues that can be jointly-concerned (through philosophical interpretation). ${ }^{6}$ In a recent elaboration of the adequacy conditions for a methodological guiding principle, a further condition, which is even more inclusive to explicitly cover those newly-developed aspect(s) of an object of study during the process of its dynamic development, has been suggested as follows ${ }^{7}$ :

\footnotetext{
${ }^{5}$ Mou 2010, 20-21.

${ }^{6}$ Indeed, to my knowledge and my first-hand research in this area, many "transparently novel" issues turn out to be distinct aspects of either "old" issues or newly identified "jointly-concerned issues" through due philosophical interpretation.

${ }^{7}$ See Mou (2014), "Introduction to Part Two: Constructive Engagement of Analytic and Continental Approaches Beyond the Western Tradition", in Bo Mou and Richard Tieszen eds (2014), Constructive Engagement of Analytic and Continental Approaches in Philosophy (Brill), 161.
} 
The dynamic-development-sensitivity condition: A methodological guiding principle is considered adequate (in this connection) if it guides the agent to be sensitive to the dynamic development (if any) of an object of study for the sake of realizing and understanding which aspects are still genuinely possessed by the object (thus which methodological perspectives are still eligible) and which ones not (thus which perspectives not eligible anymore). In contrast, it is considered inadequate in this connection if otherwise. This adequacy condition calls the agent's attention and sensitivity to this: during the process of the dynamic development (if any) of an object of study, the object might develop some new aspect(s) while losing some of its previous aspect(s); consequently, the methodological perspective with regard to the previous aspect of the object might be not absolutely or permanently eligible, and a previously ineligible perspective might become eligible because of its pointing to the new aspect. This adequacy condition highlights the need for the agent's sensitivity to the dynamic development (if any) of the object of study, one important front which can be easily ignored by an agent who is guided by an inadequate methodological guiding principle in this connection.

In this way, on the one hand, the constructive-engagement strategy does share the author's sentiment in this connection: clearly, what counts as a jointly-concerned issue in philosophy is not determined by "[being] seen before... [their] look[ing] well and truly familiar to us too".

On the other hand, it seems to me that the author's foregoing cited remarks do include some sayings that could be more refined. Indeed, there is nothing wrong with the reflective practice per se (as one "perspective" approach in philosophical exploration) of engaging some issues in the "existing" domain of inquiry; such a "perspective" approach per se constitutes one constructive and promising connection of engagement. Rather, what is "possibly" wrong lies essentially in its agents" (explicitly or implicitly) assumed "inadequate" methodological guiding principles (if any) that would treat this "perspective" approach as the exclusive one or the absolutely superior one. To this extent and in this sense, the "need" is in due place when one aims at focusing on the aspect of an object of study which such a "perspective" is to point to and capture and thus takes it to be her current working perspective. It is unclear how to understand the term 'need' in the author's phrase ' $[\mathrm{t}$ ]o gain inclusion, non-western philosophies need effectively to hook in to existing issues, interests, or paradigms in the dominant discourse' here: would it mean that one needs to indiscriminately hook in to existing issues (thus involving some "inadequate" methodological guiding principle) or that one needs to do this (as one "perspective" approach which per se does not exclude any other eligible perspectives) when one sets out to focus on such an issue? Indeed, whether or not such a need is justified really depends on the purpose of a project. I think the author could further refine these sayings here to have a more distinctive treatment of those "western" scholars who are holding the foregoing "engagement" perspective but might have distinct methodological guiding principles, some of which are adequate while some others inadequate.

3. The coverage of Anand Vaidya's article "Public Philosophy: Cross-Cultural and Multi-Disciplinary" is extensive and its contents are rich. Vaidya has suggested 
his philosophically interesting and engaging conception of the "ACE" philosophy. According to Vaidya,

....by putting together Analytical philosophy, Comparative philosophy, and Experimental, or Empirically-Engaged, philosophy we are in a better position to do public philosophy that employs a wider set of tools from what we find in any of these kinds of philosophical methods taken alone. What we have is, ACE philosophy, where 'analytical' means nothing more than analysis by reflection on cases, 'comparative' means historically informed crosscultural and cross-traditional investigation, and 'experimental or empirically-engaged' refers either to: (i) running some kind of study on a population that uses an effective instrument, not necessarily surveys; or (ii) engaging empirical work, such as what we find in cognitive science, anthropology, psychology, or linguistics." [55]

Surely there would be various types of engagement combinations in comparative philosophy understood broadly, instead of one exclusive type. The reason for Vaidya's selection of this specific engagement combination is related to his "public philosophy" concern and goal. I think such an engagement combination is well justified. On the other hand, I would like to consider how the identity of the ACE philosophy could be expanded in one substantial connection when some relevant strategic point of the constructive-engagement strategy is addressed. Let me start with one question which some of the interested readers might ask: could the ACE philosophy allow the due room for the constructive engagement between analytic and "Continental" approaches in philosophy which present two representative styles and orientation of doing philosophy and which have their distinct manifestations in some different culture-associated philosophical traditions, though currently they prominently manifest themselves in contemporary philosophy in the West? One consideration that would have such a question reasonable is this: the conception of comparative philosophy addressed in the ACE philosophy is the one in a narrow sense, i.e., that concerning only different culture-associated philosophical traditions, ${ }^{8}$ instead of a broad sense in which the constructive engagement strategy of comparative philosophy is addressed. Indeed, from the vantage point of the constructive engagement strategy of comparative philosophy, 'different philosophical traditions' does not mean only those culture-associated philosophical traditions but also different traditions associated with distinct orientations/styles of doing philosophy, such as analytic tradition and "Continental" tradition in their broad senses; that is, we talk about different/various philosophical traditions, whether distinguished culturally or by style/orientation, instead of being limited to those culturallydistinguished traditions. With the broadly understood identities of different philosophical traditions, the thus-expanded ACE philosophy could be more inclusive than what the ACE in its current identity would allow: for example, with analytic approach in philosophy as one engagement starting point, it can engage "Continental"

\footnotetext{
${ }^{8}$ As Vaidya indicates, “...by attending to cultures and traditions outside of western philosophy, ACE brings ideas and arguments into play that may have the ability to bring about a more diverse body of philosophers because the ideas are now being taken from different cultures and traditions." [56]
} 
approach in philosophy as a distinct tradition, whether these relevant "Continental" resources are from outside of or from within western philosophy. ${ }^{9}$

Vaidya delivers his ending point in the foregoing ending section this way: “...we might need to be more inclusive, so that our engagement with justice, knowledge, and beauty takes into account potentially different points of view." [57] The ending point here as a general call is worth further explanation: would "any different views" go? Would there be any criterion for this? In this connection, the constructive engagement approach has an explicit standard via its conception of eligible perspectives - only those different but "eligible" perspectives go, instead of "anything goes". Though there has yet to be a further clarification and explanation of the ending phrase 'takes into account potentially different points of view' with regard to the foregoing concern, I think that Vaidya's sample examinations of a number of concrete case analyses in the preceding discussion in his article have shown an orientation that seems to be essentially in the "eligibility" direction, instead of "any different views go". Nevertheless, theoretically speaking, I think this "criterion" issue needs to be explicitly addressed to enhance the author's general ending point. In this connection, the constructive-engagement strategy, as mentioned above, does give an answer via its conception of eligible perspectives; it expects a further engagement on this issue (including criticism of its own view on this).

Indeed, due to its foregoing general features, the constructive-engagement strategy of comparative philosophy is intrinsically open and constructive; its distinct meta-philosophical and meta-methodological part is open to further engagement and constructive dialogue, like what the three authors and the journal editor carry out through this specific topic issue. ${ }^{10}$

Bo Mou

July 2015

\footnotetext{
${ }^{9}$ For more detailed explanation in this connection, see the "Intro" etc. in Bo Mou \& Richard Tieszen eds. (2014), Constructive Engagement of Analytic and Continental Approaches in Philosophy: From the Vantage Point of Comparative Philosophy (Brill).

${ }^{10}$ I would like to express my thanks to Dean Paul Dominguez and Xiaojun Ding for their helpful suggestions on some language expressions in this writing.
} 